\title{
EULER-POINCARÉ CHARACTERISTIC AND HIGHER ORDER SECTIONAL CURVATURE. I
}

\author{
CHUAN-CHIH HSIUNG AND KENNETH MICHAEL SHISKOWSKI
}

\begin{abstract}
The following long-standing conjecture of H. Hopf is well known. Let $M$ be a compact orientable Riemannian manifold of even dimension $n \geqslant 2$. If $M$ has nonnegative sectional curvature, then the Euler-Poincare characteristic $\chi(M)$ is nonnegative. If $M$ has nonpositive sectional curvature, then $\chi(M)$ is nonnegative or nonpositive according as $n \equiv 0$ or $2 \bmod 4$. This conjecture for $n=4$ was proved first by J. W. Milnor and then by S. S. Chern by a different method. The main object of this paper is to prove this conjecture for a general $n$ under an extra condition on higher order sectional curvature, which holds automatically for $n=4$. Similar results are obtained for Kähler manifolds by using holomorphic sectional curvature, and F. Schur's theorem about the constancy of sectional curvature on a Riemannian manifold is extended.
\end{abstract}

Introduction. The first and simplest result relating local and global invariants in differential geometry is the Gauss-Bonnet formula. It expresses the Euler-Poincare characteristic $\chi(M)$ of a compact orientable Riemannian manifold $M$ of even dimension $n$ as an integral of the $n$th sectional curvature (or the Lipschitz-Killing curvature) times the volume element of $M$. Of course, for $n=2$, the $n$th sectional curvature is the usual sectional curvature.

Over the last three decades, the authors of $[5,6,9,10$, and 24] have obtained various curvature conditions determining the sign of $\chi(M)$. However, the following long-standing conjecture remains open.

H. HOPF'S CONJECTURE. If $M$ has nonnegative sectional curvature, then $\chi(M)$ is nonnegative. If $M$ has nonpositive sectional curvature, then $\chi(M)$ is nonnegative when $n \equiv 0 \bmod 4$, and nonpositive when $n \equiv 2 \bmod 4$.

This conjecture cannot be established (see [7 and 13]) just by use of the GaussBonnet formula. For $n=4$, the conjecture was proven by J. W. Milnor, and then by S. S. Chern in [5], using a different method. In [1], R. L. Bishop and S. I. Goldberg obtained a similar result for Kähler manifolds, using holomorphic sectional curvature instead of sectional curvature. Recently, D. L. Johnson [12] proved the Hopf conjecture for Kähler manifolds of real dimension 6.

Received by the editors December 5, 1983 and, in revised form, October 29, 1986. Presented to the Society, January 27, 1984, under the title Euler-Poincare characteristic.

1980 Mathematics Subject Classification (1985 Revision). Primary 53C20, 53C55, 53C65, 57R20. 
In this paper, we relate the sign of $\chi(M)$ to the sign of higher order sectional curvatures, studying higher order holomorphic sectional curvatures when $M$ is Kähler. The main results are these.

THEOREM A. Let $M$ be an oriented compact Riemannian manifold of even dimension $n$, and $p$ be an even integer with $2 \leqslant p \leqslant n$. If the pth and $(n-p)$ th curvature operators of $M$ are both nonnegative or both nonpositive, then the Euler-Poincare characteristic $\chi(M) \geqslant 0$. If one is nonnegative and the other nonpositive, then $\chi(M) \leqslant 0$.

THEOREM B. Let $M$ be a connected Kähler manifold of real dimension $2 n$ with $n \geqslant 2$, and let $p$ be an even integer with $2 \leqslant p<2 n$. If $M$ has pointwise constant pth holomorphic sectional curvature, then $M$ has constant pth holomorphic sectional curvature.

THEOREM C. Let $M$ be a Kähler manifold of real dimension $2 n$ with $n \geqslant 2$, and let $p$ be an even integer with $2 \leqslant p<2 n$. If $M$ has constant zero pth holomorphic sectional curvature, then $M$ has constant zero qth holomorphic sectional curvature for all even integers $q$ with $q \geqslant p$. If $M$ is also compact, then the Euler-Poincare characteristic $\chi(M)$ is zero.

In fact, a more general result than Theorem $A$ is proved in $\$ 2$. However, for $n=4$, Theorem A can be shown to reduce to the Hopf conjecture. Theorem $\mathrm{B}$ is well known when $p=2$. Restated for a Riemannian manifold $M$ with usual sectional curvature in place of holomorphic sectional curvature, Theorem B is the famous theorem of F. Schur, established for $2<p<2 n$ by J. R. Thorpe [24]. As originally stated, above, Theorem B was proved by R. M. Naviera [18], using methods different from ours. Theorem $\mathrm{C}$ is proved in $\S 5$. A similar result for a Riemannian $M$ was obtained by J. R. Thorpe in [24].

1. Curvature operators. Let $V$ be an $n$-dimensional real inner product space with inner product $\langle$,$\rangle . For p$ an integer, $1 \leqslant p \leqslant n$, let $\Lambda^{p}(V)$ or simply $\Lambda^{p}$ denote the space of $p$-vectors of $V$. We call a $p$-vector $\alpha$ decomposable if it can be written in the form $u_{1} \wedge \cdots \wedge u_{p}$ with $u_{i} \in V$. Any basis $E=\left\{e_{1}, \ldots, e_{n}\right\}$ for $V$ induces a basis $\left\{e_{i_{1}} \wedge \cdots \wedge e_{i_{p}} \mid 1 \leqslant i_{1}<\cdots<i_{p} \leqslant n\right\}$ for $\Lambda^{p}$ consisting of decomposable $p$-vectors. An arbitrary $p$-vector $\alpha$ is of the form

$$
\alpha=\sum \alpha_{i_{1} \ldots i_{p}} e_{i_{1}} \wedge \cdots \wedge e_{i_{p}},
$$

where summation extends over all $1 \leqslant i_{1}<\cdots<i_{p} \leqslant n$. The coefficients $\alpha_{i_{1} \cdots i_{p}}$, skew-symmetric in their indices, are called the Plücker coordinates of $\alpha$ with respect to the basis $E$. We define an inner product for $\Lambda^{p}$ on decomposable $p$-vectors by

$$
\left\langle u_{1} \wedge \cdots \wedge u_{p}, v_{1} \wedge \cdots \wedge v_{p}\right\rangle=\operatorname{det}\left[\left\langle u_{i}, v_{j}\right\rangle\right],
$$

where $u_{i}, v_{j} \in V$. It is easily seen that the basis for $\Lambda^{p}$ induced by an orthonormal basis for $V$ is itself orthonormal.

We identify the Grassmann manifold $G$ of oriented $p$-dimensional subspaces $P$ of $V$ with the submanifold of $\Lambda^{p}$ consisting of decomposable $p$-vectors of length one 
by

$$
P \Leftrightarrow u_{1} \wedge \cdots \wedge u_{p}
$$

where $\left\{u_{1}, \ldots, u_{p}\right\}$ is an oriented orthonormal basis for $P$. Elements of $G$ will be called $p$-planes.

The following result is well known and will be needed later. For a proof, see Hodge and Pedoe [11, pp. 309ff].

Lemma 1.1 (The Grassmann Quadratic p-Relations). Choose a basis $\left\{e_{1}, \ldots, e_{n}\right\}$ for $V$. Then the p-vector $\alpha$ is decomposable if and only if its Plücker coordinates satisfy

$$
\sum_{k=1}^{p+1}(-1)^{k} \alpha_{i_{1} \ldots i_{k} \cdots i_{p+1}} \alpha_{i_{k} j_{1} \cdots j_{p-1}}=0,
$$

for all $1 \leqslant i_{k}, j_{l} \leqslant n$ where the symbol ^over $i_{k}$ indicates that the subscript $i_{k}$ is to be deleted.

Let $\mathscr{R}^{p}$, or simply $\mathscr{R}$ for fixed $p$, denote the vector space of all selfadjoint linear transformations on $\Lambda^{p}$ with inner product $\langle T, U\rangle=\operatorname{trace}(T \circ U)$. Elements of $\mathscr{R}$ are called $p$ th (order) curvature operators on $V$. We associate to each $R \in \mathscr{R}$ its $p$ th (order) sectional curvature function $\sigma_{R}: G \rightarrow \mathbf{R}$ defined by $\sigma_{R}(P)=\langle R(P), P\rangle$ for all $P \in G$, where $\mathbf{R}$ is the set of real numbers. Then $R_{p} \in \mathscr{R}^{p}$ satisfies the Bianchi identities if

(2) $\sum_{k=1}^{p+1}\left\langle(-1)^{k} R_{p}\left(u_{1} \wedge \cdots \wedge \hat{u}_{k} \wedge \cdots \wedge u_{p+1}\right), u_{k} \wedge u_{p+2} \wedge \cdots \wedge u_{2 p}\right\rangle=0$,

for all $u_{1}, \ldots, u_{2 p} \in V$. Let $\mathscr{B}$ denote the space of elements of $\mathscr{R}$ which satisfy the Bianchi identities, and let $\mathscr{S}$ denote the space of elements $R \in \mathscr{R}$ for which $\sigma_{R} \equiv 0$. Then from Stehney $[23, \S 1]$ we have the following.

LEMMA 1.2. With respect to the inner product defined above on $\mathscr{R}, \mathscr{R}=\mathscr{B} \oplus \mathscr{S}$ is an orthogonal decomposition of $\mathscr{R}$.

We will need the following consequence of Lemma 1.2.

LeMma 1.3. For $p$ an integer with $0 \leqslant p \leqslant n$, let ${ }^{*} p: \Lambda^{p} \rightarrow \Lambda^{n-p}$ be the Hodge star operator. Then the map $\Omega: \mathscr{R}^{p} \rightarrow \mathscr{R}^{n-p}$ is orthogonal with respect to the above inner product where $\Omega(S)={ }^{*} p S^{*}(n-p)$ for all $S \in \mathscr{R}^{p}$.

Proof. First, $\Omega$ is well defined since the adjoint of ${ }^{*} p$ is

$$
\left({ }^{*} p\right)^{-1}=(-1)^{p(n-p)} *(n-p)
$$

Thus for $S, T \in \mathscr{R}^{p}$ we have

$$
\begin{aligned}
\left\langle{ }^{*} p S^{*}(n-p),{ }^{*} p T^{*}(n-p)\right\rangle & =\operatorname{trace}\left({ }^{*} p S^{*}(n-p) \circ{ }^{*} p T^{*}(n-p)\right) \\
& =\operatorname{trace}\left((-1)^{p(n-p)} * p(S \circ T) *(n-p)\right) \\
& =\operatorname{trace}\left({ }^{*} p(S \circ T)\left({ }^{*} p\right)^{-1}\right) \\
& =\operatorname{trace}(S \circ T)=\langle S, T\rangle .
\end{aligned}
$$

Hence the map $\Omega$ is orthogonal. 
COROllaRy 1.4. The map $\Omega: \mathscr{R}^{p} \rightarrow \mathscr{R}^{n-p}$ preserves the orthogonal decomposition given in Lemma 1.2, i.e., $\Omega$ preserves the Bianchi identities as well as the operators with identically zero sectional curvature.

Proof. In order to show that $\Omega$ preserves the orthogonal decomposition, it is sufficient to show that for all $S \in \mathscr{S}^{p},{ }^{*} p S^{*}(n-p) \in \mathscr{S}^{n-p}$ since we know by Lemma 1.3 that $\Omega$ is orthogonal. Now for an $(n-p)$-plane $\alpha$ in $V, *(n-p)(\alpha)$ is a $p$-plane in $V$. Thus for all $S \in \mathscr{S}^{p}$,

$$
\left\langle{ }^{*} p S^{*}(n-p)(\alpha), \alpha\right\rangle=(-1)^{p(n-p)}\left\langle S\left({ }^{*} n-p\right) \alpha,{ }^{*}(n-p) \alpha\right\rangle=0,
$$

so that ${ }^{*} p S^{*}(n-p) \in \mathscr{S}^{n-p}$.

Although the statements above are true in a general inner product space setting, our main interest is the $p$ th curvature operator arising on a tangent space $M_{m}$ of a Riemannian manifold $M$ at a point $m$. Specifically, let $R$ denote the Riemannian curvature tensor at $m$. Then for even integers $p \geqslant 2$, the $p$ th curvature operator $R_{p}$ is defined in Thorpe [26] by

$$
\begin{aligned}
&\left\langleR _ { p } \left( u_{1} \wedge \cdots\right.\right.\left.\left.\wedge u_{p}\right), v_{1} \wedge \cdots \wedge v_{p}\right\rangle \\
&=\frac{1}{2^{p / 2} p !} \sum_{\alpha, \beta \in S_{p}} \operatorname{sgn}(\alpha) \operatorname{sgn}(\beta) R\left(u_{\alpha(1)}, u_{\alpha(2)}, v_{\beta(1)}, v_{\beta(2)}\right) \\
& \cdots R\left(u_{\alpha(p-1)}, u_{\alpha(p)}, v_{\beta(p-1)}, v_{\beta(p)}\right),
\end{aligned}
$$

where $u_{i}, v_{j} \in M_{m}$, and $S_{p}$ is the permutation group on $(1, \ldots, p)$. Also, Thorpe [26] establishes that $R_{p}$ satisfies the Bianchi identities.

The generalized Gauss-Bonnet theorem expresses the Euler-Poincare characteristic $\chi(M)$ of a compact oriented Riemannian manifold $M$ of even dimension $n$ as the integral

$$
\chi(M)=\frac{2}{c_{n}} \int_{M} K d V
$$

where $K$ is the Lipschitz-Killing curvature of $M, c_{n}$ is the volume of the Euclidean unit $n$-sphere, and $d V$ is the volume element of $M$.

The following useful theorem is a composite of the two theorems and Corollary 2 from Thorpe [26]. We write $\sigma_{2 k}$ for $\sigma_{R_{2 k}}$.

THEOREM 1.5. Let $M$ be an oriented Riemannian manifold of even dimension $n$. Then we have the following.

(a) The Lipschitz-Killing curvature $K$ at $m \in M$ satisfies

$$
K(m)=\left\langle R_{n}\left(M_{m}\right), M_{m}\right\rangle=\frac{p !(n-p) !}{n !} \operatorname{trace}\left(*(n-p) R_{n-p}{ }^{*} p R_{p}\right) .
$$

(b) Let $n=4 k$. Then $R_{2 k}{ }^{*}(2 k)= \pm{ }^{*}(2 k) R_{2 k}$ if and only if $\sigma_{2 k}\left({ }^{*}(2 k) P\right)=$ $\pm \sigma_{2 k} P$ for all $2 k$-planes $P$. Signs above are to be taken consistently, with plus implying that $K \geqslant 0$, minus that $K \leqslant 0$.

(c) Let $M$ be compact with $n=4 k, R_{2 k}= \pm{ }^{*}(2 k) R_{2 k}$, and $P_{k}$ denoting the $k$ th Pontryagin class of $M$. Then the plus sign gives

$$
\chi(M) \geqslant \frac{k ! k !}{(2 k) !}\left|P_{k}\right| \geqslant 0,
$$


while the minus sign gives $\chi(M) \leqslant 0$ and $P_{k}=0$. Moreover, $\chi(M)=0$ if and only if $M$ is $2 k$-flat, i.e., $\sigma_{2 k} \equiv 0$.

Now we consider the case of a Kähler manifold $M$ with an almost complex structure $J$ and Riemannian curvature operator $R$.

Definition 1.6. Also denote by $J$ the extension of the structure $J$ from $M_{m}$ to $\Lambda^{p}\left(M_{m}\right)$ defined by $J\left(u_{1} \wedge \cdots \wedge u_{p}\right)=J u_{1} \wedge \cdots \wedge J u_{p}$ for $u_{i} \in M_{m}$, extending linearly to $\Lambda^{p}\left(M_{m}\right)$. Note that this gives $J^{2}=(-1)^{p}$.

Lemma 1.7. (a) Let $R_{p}$ be the pth curvature operator on $M$. Then $R_{p}=R_{p} \circ J=$ $J \circ R_{p}$.

(b) The adjoint of $J$ is $(-1)^{p} J$.

(c) For even $p, J$ and ${ }^{*} p$ commute.

Proof. (a) This follows directly from the definition of $R_{p}$ and the fact that for a Kähler manifold $R=R \circ J=J \circ R$.

(b) This follows directly from the definitions of $\langle$,$\rangle and J$ on $\Lambda^{p}\left(M_{m}\right)$, and the fact that the adjoint of $J$ on $M_{m}$ is $-J$.

(c) Let $p$ be even. Now the Hodge star operator ${ }^{*} p: \Lambda^{p}\left(M_{m}\right) \rightarrow \Lambda^{n-p}\left(M_{m}\right)$ can be defined by

$$
\left({ }^{*} p \xi\right) \wedge \eta=\langle\xi, \eta\rangle M_{m},
$$

for $\xi, \eta \in \Lambda^{p}\left(M_{m}\right)$, where $M_{m}$ is the oriented $n$-plane which is the tangent space of $M$ at $m$. Let $\xi, \eta \in \Lambda^{p}\left(M_{m}\right)$. Since $J M_{m}=M_{m}$ and the adjoint of $J$ is $J$, we have

$$
\begin{aligned}
{\left[\left(J^{*} p J\right)(\xi)\right] \wedge \eta } & =J\left(\left[{ }^{*} p(J \xi)\right] \wedge J \eta\right) \\
& =J\left(\langle J \xi, J \eta\rangle M_{m}\right) \\
& =\langle\xi, \eta\rangle M_{m} .
\end{aligned}
$$

Hence $J^{*} p J={ }^{*} p$, which implies ${ }^{*} p J=J^{*} p$.

Definition 1.8. For $p$ even, a $p$-plane $Q$ is said to be holomorphic if $J Q=Q$. Also, $p$ th holomorphic sectional curvature is $p$ th sectional curvature restricted to holomorphic $p$-planes.

THEOREM 1.9. Let $V$ be an even-dimensional real inner product space with a complex structure $J$ and Hermitian inner product $\langle$,$\rangle . Also, let T: \Lambda^{2}(V) \rightarrow \Lambda^{2}(V)$ be a self-adjoint linear operator satisfying the Bianchi identity and $T=T \circ J=J \circ T$. Then we have the following.

(a) $T$ is uniquely determined by its holomorphic sectional curvature, i.e., if $T$ has zero holomorphic sectional curvature, then $T \equiv 0$.

(b) If $T$ has constant holomorphic sectional curvature $H$, then for all $X, Y, Z, W \in V$ we have

$$
\begin{aligned}
\langle T(X \wedge Y), Z \wedge W\rangle & \\
=(H / 4)[\langle X, Z\rangle,\langle Y, W\rangle- & \langle X, W\rangle\langle Y, Z\rangle+\langle X, J Z\rangle\langle Y, J W\rangle \\
& -\langle X, J W\rangle\langle Y, J Z\rangle+\langle X, J Y\rangle\langle Z, J W\rangle] .
\end{aligned}
$$


Proof. This theorem is merely a restatement of Kobayashi-Nomizu [14, vol. II, Chapter IX, Propositions 7.1 and 7.3].

COROLlaRY 1.10. Let $V$ and $T$ satisfy the hypotheses of Theorem 1.9 with $\operatorname{dim} V=2 n$ and $T$ having constant holomorphic sectional curvature $H$. Let $\left\{e_{1}, e_{1^{*}}, \ldots, e_{n}, e_{n^{*}}\right\}$ be an orthonormal basis of $V$, where $e_{i^{*}}=J e_{i}$ for $i=1, \ldots, n$. Then from this basis of $V$, we have an orthonormal basis $B$ of $\Lambda^{2}(V)$ given by $B=\left\{e_{i_{1}} \wedge e_{i_{2}} \mid 1 \leqslant i_{1}<i_{2} \leqslant n^{*}\right.$ where $\left.1<1^{*}<\cdots<n<n^{*}\right\}$. Furthermore, for $Q \in B$ we have

$$
T(Q)=\left\{\begin{array}{cl}
H Q+\frac{H}{2} \sum_{\substack{Q^{\prime} \in B, Q^{\prime} \neq Q \\
Q^{\prime} \text { holomorphic }}} Q^{\prime}, & \text { if } Q \text { is holomorphic, } \\
\frac{1}{4} H(Q+J Q), & \text { if } Q \text { is not holomorphic. }
\end{array}\right.
$$

Proof. This obviously follows from (5) and the fact that $B$ is an orthonormal basis of $\Lambda^{2}(V)$.

Finally, we mention the relationship between the $k$ th Chern form and the $(2 k)$ th curvature operator on a compact Kähler manifold given in $\$ 2$ of Gray [10].

THEOREM 1. Let $M$ be a compact Kähler manifold of real dimension $2 n$ with almost complex structure J. For $m \in M$, let $\left\{e_{1}, e_{1^{*}}, \ldots, e_{n}, e_{n^{*}}\right\}$ be an orthonormal basis of $M_{m}$ where $e_{i *}=J e_{i}$ for $i=1, \ldots, n$, let $\gamma_{k}$ be the kth Chern form (via de Rham's theorem), and let $R_{2 k}$ be the ( $\left.2 k\right)$ th curvature operator. Then

$$
\begin{aligned}
\frac{(4 \pi)^{k} k !}{(2 k) !} & \gamma_{k}\left(j_{1}, \ldots, j_{2 k}\right) \\
& =\sum_{1 \leqslant i_{1}<\cdots<i_{k} \leqslant n}\left\langle R_{2 k}\left(e_{i_{1}} \wedge e_{i_{1}^{*}} \wedge \cdots \wedge e_{i_{k}} \wedge e_{i_{k}^{*}}\right), j_{1} \wedge \cdots \wedge j_{2 k}\right\rangle,
\end{aligned}
$$

for all $j_{1}, \ldots, j_{2 k} \in M_{m}$.

Proof. This follows directly from (2.4), Lemma 2.1, the definition of $(2 k)$ th curvature operator in $\S 2$ of Gray [10], and the fact that Gray's $(2 k)$ th curvature operator is $\left[(2 k) ! / 2^{k}\right] R_{2 k}$.

2. Euler-Poincaré characteristic and curvature operators. Let $\Pi_{p}: \mathscr{R}^{p} \rightarrow \mathscr{B}^{p}$ be the orthogonal projection associated with the orthogonal decomposition of Lemma 1.2 .

THEOREM 2.1. Let $M$ be an oriented compact Riemannian manifold of even dimension $n$, and $p$ be an even integer with $2 \leqslant p<n$. Let $R_{p}$ and $R_{n-p}$ be the pth and $(n-p)$ th curvature operators on $M$. If at each $m \in M$ there exists $\bar{R}_{p} \in \mathscr{R}^{p}$ such that $\Pi_{p} \bar{R}_{p}=R_{p}$ with $\bar{R}_{p}$ and $R_{n-p}$ both positive semidefinite or negative semidefinite operators, then the Euler-Poincare characteristic $\chi(M) \geqslant 0$. If semidefinite is replaced by definite at at least one point of $M$, then $\chi(M)>0$. If $\bar{R}_{p}$ and $R_{n-p}$ are of opposite signs as operators, then $\chi(M) \leqslant 0$ or $\chi(M)<0$ according as both $\vec{R}_{p}$ and $R_{n-p}$ are semidefinite or definite (at at least one point of $M$ ). 
Proof. Assume that at each $m \in M$ the above holds with operators $\bar{R}_{p}$ and $R_{n-p}$ both positive semidefinite. The other cases can be proven similarly. Since $\Pi_{p} \bar{R}_{p}=$ $R_{p}$, there exists $S_{p} \in \mathscr{S}^{p}$ with $\bar{R}_{p}=R_{p}+S_{p}$.

Let $\left\{v_{1}, \ldots, v_{k}\right\}$ be an orthonormal basis of $\Lambda^{p}\left(M_{m}\right)$ consisting of eigenvectors of $\bar{R}_{p}$, and let $\left\{\lambda_{1}, \ldots, \lambda_{k}\right\}$ be the corresponding eigenvalues of $\bar{R}_{p}$ with respect to the given basis. Let $K(m)$ be the Lipschitz-Killing curvature of $M$ at $m$. Then by Corollary 1.4 , Lemma 1.2 , and (4) we have

$$
\begin{aligned}
K(m) & =[p !(n-p) ! / n !] \operatorname{trace}\left(\left[*(n-p) R_{n-p}{ }^{*} p\right]\left[\bar{R}_{p}-S_{p}\right]\right) \\
& =[p !(n-p) ! / n !]\left[\operatorname{trace}\left(*(n-p) R_{n-p}{ }^{*} p \bar{R}_{p}\right)-\operatorname{trace}\left(*(n-p) R_{n-p}{ }^{*} p S_{p}\right)\right] \\
& =[p !(n-p) ! / n !] \operatorname{trace}\left(*(n-p) R_{n-p}{ }^{*} p \bar{R}_{p}\right) \\
& =[p !(n-p) ! / n !] \sum_{i=1}^{k}\left\langle *(n-p) R_{n-p}{ }^{*} p \bar{R}_{p}\left(v_{i}\right), v_{i}\right\rangle \\
& =[p !(n-p) ! / n !] \sum_{i=1}^{k} \lambda_{i}\left\langle *(n-p) R_{n-p}{ }^{*} p\left(v_{i}\right), v_{i}\right\rangle \\
& =[p !(n-p) ! / n !] \sum_{i=1}^{k} \lambda_{i}\left\langle R_{n-p}\left({ }^{*} p v_{i}\right),{ }^{*} p v_{i}\right\rangle \geqslant 0
\end{aligned}
$$

since $\lambda_{i} \geqslant 0$ for $i=1, \ldots, k$, and $R_{n-p}$ is positive semidefinite gives $\left\langle R_{n-p}(\alpha)\right.$, $\alpha\rangle \geqslant 0$ for all $\alpha \in \Lambda^{n-p}$. Hence by the generalized Gauss-Bonnet formula (3) we have $\chi(M) \geqslant 0$.

REMARK 2.2. (a) Let the dimension of $V$ be four. Then Thorpe [27] has shown that $R \in \mathscr{B}^{2}$ has nonnegative (positive) sectional curvature if and only if $R=\Pi_{2} \bar{R}$ for some positive semidefinite (definite) $\bar{R} \in \mathscr{R}^{2}$.

(b) D. Meyer [16] has shown that a compact Riemannian manifold with positive definite 2 nd (usual) curvature operator is a homology sphere.

(c) Thorpe [24] characterizes the $p$ th curvature operators $R_{p}$ of constant $p$ th sectional curvature $K$ as $R_{p}=K \cdot I$ where $I$ is the identity operator. These are positive or negative definite according as $K>0$ or $K<0$.

3. Curvature operators of the form $R_{p}=c A^{p}$ on a Kähler manifold. Following Stehney [22] we can define a selfadjoint operator $R_{p}=c A^{p}$ on $\Lambda^{p}(V)$ for $c \in \mathbf{R}$ and a selfadjoint operator $A$ on $V$ by $c A^{p}\left(u_{1} \wedge \cdots \wedge u_{p}\right)=c\left(A u_{1} \wedge \cdots \wedge A u_{p}\right)$ for all $u_{i} \in V$, extending linearly to $\Lambda^{p}(V)$. Moreover, $R_{p}=c A^{p}$ satisfies the Bianchi identities.

THEOREM 3.1. Let $V$ be a real inner product space of dimension $2 n$, and $J$ be a complex structure on $V$. For $p$ an even integer with $2 \leqslant p<2 n$, let $R_{p}=c A^{p}$ be an operator on $\Lambda^{p}(V)$ as defined above with $A^{p}=J \circ A^{p}=A^{p} \circ J$. Also, let $\left\{v_{1}, \ldots, v_{2 n}\right\}$ be an orthonormal basis of $V$ consisting of eigenvectors of $A$ with corresponding eigenvalues $\lambda_{1}, \ldots, \lambda_{2 n}$. Then either $R_{p} \equiv 0$ or $A$ has exactly $p$ nonzero eigenvalues $\lambda_{i_{1}}, \ldots, \lambda_{i_{p}}\left(1 \leqslant i_{1}<\cdots<i_{p} \leqslant 2 n\right)$ with $v_{i_{1}} \wedge \cdots \wedge v_{i_{p}}$ a holomorphic 
p-plane, so that for all $\gamma \in \Lambda^{p}(V)$

$$
R_{p}(\gamma)=c \lambda_{i_{1}} \cdots \lambda_{i_{p}}\left\langle\gamma, v_{i_{1}} \wedge \cdots \wedge v_{i_{p}}\right\rangle v_{i_{1}} \wedge \cdots \wedge v_{i_{p}} .
$$

Proof. Let $B=\left\{v_{j_{1}} \wedge \cdots \wedge v_{j_{p}} \mid 1 \leqslant j_{1}<\cdots<j_{p} \leqslant 2 n\right\}$ for the above orthonormal basis of $\Lambda^{p}(V)$ consisting of eigenvectors of $R_{p}$ with corresponding eigenvalues $c \lambda_{j_{1}} \cdots \lambda_{j_{p}}$.

Assume that $R_{p}$ is not identically zero. Then $c \neq 0$ and there exist $1 \leqslant i_{1}<\cdots$ $<i_{p} \leqslant 2 n$ so that $\lambda_{i_{1}} \cdots \lambda_{i_{p}} \neq 0$. Thus $A$ has nonzero eigenvalues $\lambda_{i_{1}}, \ldots, \lambda_{i_{p}}$ with $1 \leqslant i_{1}<\cdots<i_{p} \leqslant 2 n$. Also, the corresponding eigenvector $v_{i_{1}} \wedge \cdots \wedge v_{i_{p}}$ of $R_{p}$ is a holomorphic $p$-plane since $R_{p}=J \circ R_{p}$.

Now assume there exists $\lambda_{j} \neq 0$ for some $j \neq i_{1}, \ldots, i_{p}$. Then $\lambda_{i_{1}} \cdots \lambda_{i_{p-1}} \lambda_{j}$ is not zero, and $v_{i_{1}} \wedge \cdots \wedge v_{i_{p-1}} \wedge v_{j}$ is an eigenvector of $R_{p}$ with eigenvalue $\lambda_{i_{1}} \cdots$ $\lambda_{i_{p-1}} \lambda_{j}$. Since $R_{p}=J \circ R_{p}, v_{i_{1}} \wedge \cdots \wedge v_{i_{p-1}} \wedge v_{j}$ is also a holomorphic $p$-plane. Now $v_{i_{1}} \wedge \cdots \wedge v_{i_{p}}$ and $v_{i_{1}} \wedge \cdots \wedge v_{i_{p-1}} \wedge v_{j}$ are two holomorphic $p$-planes which intersect in a holomorphic $(p-1)$-plane. This is a contradiction since $p-1$ is odd. Hence $\lambda_{j}=0$ for all $j \neq i_{1}, \ldots, i_{p}$.

Thus all other eigenvalues of $R_{p}$ are zero other than $c \lambda_{i_{1}} \cdots \lambda_{i_{p}}$, and $R_{p}$ must be of the stated form since $B$ is an orthonormal basis of $\Lambda^{p}(V)$.

Corollary 3.2. Let $M$ be a Kähler manifold of real dimension $2 n$. For $p$ an even integer with $2 \leqslant p<2 n$, if the pth sectional curvature of $M$ at $m \in M$ is constant at value $K$, then $K=0$.

Proof. Assume the above is given with $K \neq 0$. Then from Thorpe [24], the $p$ th curvature operator $R_{p}$ at $m$ is of the form $R_{p}=K \cdot I^{p}$ for the identity $I$. This contradicts Theorem 3.1 since $I$ has $2 n$ nonzero eigenvalues. Hence, $K=0$.

COROllary 3.3. Let $M$ be a compact Kähler manifold of real dimension $2 n$. If at each $m \in M$ there exists $c \in \mathbf{R}$, an even integer $p$ with $2 \leqslant p \leqslant n$, and a selfadjoint operator $A$ on $M_{m}$ so that the pth curvature operator $R_{p}$ of $M$ at $m \in M$ is of the form $R_{p}=c A^{p}$, then the Euler-Poincare characteristic $\chi(M)=0$, and the nth Chern class $\gamma_{n}$ of $M$ is zero.

Proof. Let the above at $m \in M$ be given. Then at $m \in M$, the $(2 p)$ th curvature operator $R_{2 p}=c^{2} A^{2 p}$ by Lemma 2.5 of Stehney [22]. In the following we assume $R_{p}$ is written in the form of Theorem 3.1.

CASE 1 . Let $2 \leqslant p<n$. Then $2<2 p<2 n$ and Theorem 3.1 imply that $R_{2 p} \equiv 0$ at $m \in M$, since $A$ cannot have both exactly $p$ and $2 p$ nonzero eigenvalues. Hence by the trace formula (4) for the Lipschitz-Killing curvature $K$ at $m$, we have $K(m)=0$. Also, (4) gives $R_{2 n}(m)=0$.

CASE 2. Let $p=n$. Then $R_{2 n}=c^{2} A^{2 n}=\left(c^{2} \operatorname{det} A\right) I^{2 n}$ at $m \in M$ for the identity I. But det $A=0$ since $A$ has exactly $n$ nonzero eigenvalues or we must have $c=0$ by Theorem 3.1. For either situation, we have $R_{2 n} \equiv 0$ at $m$, and formula (4) again gives $K(m)=0$.

Now since the above occurs at each $m \in M, K \equiv 0$ and $R_{2 n} \equiv 0$ on $M$. Hence the generalized Gauss-Bonnet formula (3) gives $\chi(M)=0$, and formula (7) implies that $\gamma_{n}$ is zero also. 
COROllaRY 3.4. Let the hypothesis of Corollary 3.3 be given with p constant on $M$. Then the kth Chern classes $\gamma_{k}$ of $M$ are zero for $k \geqslant p$, and $\gamma_{p / 2}$ is zero if and only if $M$ is p-flat. Also, $M$ is q-flat for all $q \geqslant 2 p$.

Proof. From the proof of Corollary 3.3 it follows that the $(2 p)$ th curvature operator $R_{2 p} \equiv 0$ on $M$. Thus the $q$ th curvature operator $R_{q} \equiv 0$ for all $q \geqslant 2 p$ by Thorpe [24, Theorem 6.4] and [26, Lemma of §3]. Hence we have by formula (7) that $\gamma_{k}$ is zero for $k \geqslant p$. Also, $R_{q} \equiv 0$ on $M$ for all $q \geqslant 2 p$ implies that $M$ is $q$-flat for all $q \geqslant 2 p$.

Now if $M$ is $p$-flat, then the above Lemma of Thorpe [26] gives $R_{p} \equiv 0$ on $M$, and formula (7) implies that $\gamma_{p / 2}$ is zero.

On the other hand, let $\gamma_{p / 2}$ be zero and assume $M$ is not $p$-flat. Suppose at some $m_{0} \in M, R_{p}$ is not identically zero and is of the nonzero form stated in Theorem 3.1. In the notation of Theorem 3.1, the holomorphic $p$-plane $v_{i_{1}} \wedge \cdots \wedge v_{i_{p}}$ can be written as $e_{1} \wedge e_{1^{*}} \wedge \cdots \wedge e_{r} \wedge e_{r^{*}}$ where $r=p / 2$ for an orthonormal basis $\left\{e_{1}, e_{1^{*}}, \ldots, e_{n}, e_{n^{*}}\right\}$ of $M_{m_{0}}$ with $e_{j^{*}}=J e_{j}$ for all $j=1, \ldots, n$. Now using formula (7) and Theorem 3.1 we get at $m_{0}$ that

$$
\begin{aligned}
0 & =(r !)(2 \pi)^{r} \gamma_{p / 2}\left(e_{1}, e_{1^{*}}, \ldots, e_{r}, e_{r^{*}}\right) \\
& =\frac{(2 r) !}{2^{r}} \sum_{1 \leqslant j_{1}<\cdots<j_{r} \leqslant n}\left\langle R_{p}\left(e_{j_{1}} \wedge e_{j_{1}^{*}} \wedge \cdots \wedge e_{j_{r}} \wedge e_{j_{r}^{*}}\right),\right. \\
& \left.e_{1} \wedge e_{1^{*}} \wedge \cdots \wedge e_{r} \wedge e_{r^{*}}\right\rangle \\
& =\frac{(2 r) !}{2^{r}} c \lambda_{i_{1}} \cdots \lambda_{i_{p}} .
\end{aligned}
$$

But $c \lambda_{i_{1}} \cdots \lambda_{i_{p}} \neq 0$ by Theorem 3.1. Thus we have a contradiction, and hence $M$ is p-flat.

COROllary 3.5. Let the hypothesis of Corollary 3.3 be given with p constant on $M$ and $n<p<2 n$. If the $(2 n-p)$ th curvature operator $R_{2 n-p}$ of $M$ has constant zero holomorphic sectional curvature, then the Euler-Poincaré characteristic $\chi(M)=0$, and the nth Chern class $\gamma_{n}$ is zero.

Proof. If $R_{p}=0$ at $m \in M$, then by formula (4) the Lipschitz-Killing curvature at $m$ is $K(m)=0$.

Also, if $R_{p} \neq 0$ at $m \in M$, then Theorem 3.1, together with its notation and proof, gives that for all $\Lambda^{p}\left(M_{m}\right)$,

$$
R_{p}(\gamma)=c \lambda_{i_{1}} \cdots \lambda_{i_{p}}\left\langle\gamma, v_{i_{1}} \wedge \cdots \wedge v_{i_{p}}\right\rangle v_{i_{1}} \wedge \cdots \wedge v_{i_{p}} .
$$

Now using formula (4) and the basis of $M_{m}$ in Theorem 3.1 we have

$$
\begin{aligned}
& {[(2 n) ! /(p !(2 n-p) !)] K(m)=\operatorname{trace}\left(*(2 n-p) R_{2 n-p}{ }^{*} p R_{p}\right)} \\
& \quad=\sum_{1 \leqslant j_{1}<\cdots<j_{p} \leqslant 2 n}\left\langle{ }^{*}(2 n-p) R_{2 n-p}{ }^{*} p R_{p}\left(v_{j_{1}} \wedge \cdots \wedge v_{j_{p}}\right), v_{j_{1}} \wedge \cdots \wedge v_{j_{p}}\right\rangle \\
& \quad=c \lambda_{i_{1}} \cdots \lambda_{i_{p}}\left\langle R_{2 n-p}\left({ }^{*} p\left[v_{i_{1}} \wedge \cdots \wedge v_{i_{p}}\right]\right),{ }^{*} p\left[v_{i_{1}} \wedge \cdots \wedge v_{i_{p}}\right]\right\rangle .
\end{aligned}
$$


Then $K(m)=0$ since $R_{2 n-p}$ has constant zero holomorphic sectional curvature, and ${ }^{*} p\left(v_{i_{1}} \wedge \cdots \wedge v_{i_{p}}\right)$ is a holomorphic $(2 n-p)$-plane by Theorem 3.1 and Lemma $1.7(\mathrm{c})$.

Thus $K \equiv 0$ on $M$, and $R_{2 n} \equiv 0$ on $M$ by formula (4). Hence the generalized Gauss-Bonnet formula (3) gives $\chi(M)=0$, and formula (7) implies that $\gamma_{n}$ is zero.

4. Constancy of holomorphic sectional curvature. The purpose of this section is to prove the following theorem.

THEOREM 4.1. Let $M$ be a connected Kähler manifold of real dimension $2 n$ with $n \geqslant 2$, and let $p$ be an even integer with $2 \leqslant p<2 n$. If $M$ has pointwise constant pth holomorphic sectional curvature, then $M$ has constant pth holomorphic sectional curvature.

Before we prove Theorem 4.1, we need the following lemma.

LemMa 4.2. Let $V$ be a real inner product space of dimension $2 n$ for $n \geqslant 2$ with complex structure $J$ and Hermitian inner product with respect to $J$. For an even integer $p$ with $2 \leqslant p<2 n$, let $\left\{e_{1}, e_{1^{*}}, \ldots, e_{n}, e_{n^{*}}\right\}$ be an orthonormal basis of $V$ with $e_{i^{*}}=J e_{i}$ for $i=1, \ldots, n$, and let $B=\left\{e_{i_{1}} \wedge \cdots \wedge e_{i_{p}} \mid 1 \leqslant i_{1}<\cdots<i_{p} \leqslant n^{*}\right\}$. Also, let $T: \Lambda^{p}(V) \rightarrow \Lambda^{p}(V)$ be a selfadjoint operator, satisfying the Bianchi identities $T=J \circ T=T \circ J$, with constant holomorphic sectional curvature $H$. Then for any holomorphic p-plane $Q \in B$,

$$
\left\langle T(Q), Q^{\prime}\right\rangle= \begin{cases}H & \text { if } Q^{\prime}=Q \\ \frac{1}{2} H & \text { if } Q^{\prime} \text { is holomorphic, and } Q^{\prime} \cap Q \text { is a } \\ & \text { holomorphic }(p-2) \text {-plane } \\ 0 \quad & \text { if } Q^{\prime} \text { is not holomorphic, but } Q^{\prime} \cap Q \\ & \text { contains a holomorphic }(p-2) \text {-plane }\end{cases}
$$

Proof. The case when $p=2$ easily follows from Corollary 1.10 , and so in the remainder of the proof we assume that $2<p<2 n$.

For $2 k=p-2$ and any $1 \leqslant j_{1}<\cdots<j_{k} \leqslant n$, let $A=e_{j_{1}} \wedge e_{j_{1^{*}}} \wedge \cdots \wedge e_{j_{k}} \wedge$ $e_{j_{k}^{*}}$ be the holomorphic $(p-2)$-plane formed from the given basis of $V$ stated above. Then $A^{\perp}$ is a holomorphic $(2 n-2 k)$-plane since the inner product is Hermitian. Now define a second-order curvature operator $R$ on $A^{\perp}$ by

$$
\langle R(X \wedge Y), Z \wedge W\rangle=\langle T(A \wedge X \wedge Y), A \wedge Z \wedge W\rangle
$$

for all $X, Y, Z, W \in A^{\perp}$, extending linearly. Then $R$ satisfies the hypothesis of Corollary 1.10, and using formula (6) with the basis of $A^{\perp}$ taken from the given basis of $V$ we easily get formula (8) for $T$.

ProOF OF THEOREM 4.1. Let $F(M)$ be the principal $O(2 n)$-bundle of orthonormal frames on $M$, where $O(2 n)$ is the group of orthogonal $2 n \times 2 n$ matrices. Let $\Pi$ : $F(M) \rightarrow M$ be the projection map.

The curvature form $\Omega=\left[\Omega_{i j}\right]$ of the Riemannian connection of $M$ is a smooth 2-form on $F(M)$ with values in the Lie algerbra $o(2 n)$ of real skew-symmetric $2 n \times 2 n$ matrices. If $v, w$ are tangent vectors at $z=\left(m ; e_{1}, \ldots, e_{2 n}\right) \in F(M)$, with 
$R$ the Riemannian curvature operator, we have for $v^{\prime}=\Pi_{*}(v)$ and $w^{\prime}=\Pi_{*}(w)$ that $\Omega_{i j}(z)(v, w)=\left\langle R\left(e_{i} \wedge e_{j}\right), v^{\prime} \wedge w^{\prime}\right\rangle$.

For $I=\left\{i_{1}, \ldots, i_{p}\right\}$ with $i_{1}, \ldots, i_{p}$ integers between 1 and $2 n$, we define

$$
\theta_{I}=(1 / p !) \sum_{J} \delta_{J}^{I} \Omega_{j_{\nu} j_{2}} \wedge \cdots \wedge \Omega_{j_{p-1} j_{p}},
$$

where $J=\left\{j_{1}, \ldots, j_{p}\right\}$ for $j_{1}, \ldots, j_{p}$ integers between 1 and $2 n$, with $\delta_{J}^{I}=1$ (or -1 ) if the integers $i_{1}, \ldots, i_{p}$ are distinct and $J$ is an even (or odd) permutation of $I$, with $\delta_{J}^{1}=0$ otherwise, and with the sum $\sum_{J}$ taken over all selections $J$ from $\{1, \ldots, 2 n\}$. Then $\theta_{I}$ is a $p$-form on $F(M)$ for all $I$. Also $D \theta_{I}=0$ for all $I$ since $D \Omega=0$ where $D$ is covariant differentiation. Specifically, $D \alpha=d \alpha \circ h$ for an arbitrary form $\alpha$ where $d$ is exterior differentiation and $h$ takes the horizontal part.

The 1-forms $\omega_{i}$ are defined on $F(M)$ for $i=1, \ldots, 2 n$ and a tangent vector $v$ at $z=\left(m ; e_{1}, \ldots, e_{2 n}\right) \in F(M)$ by $\omega_{i}(z)(v)=\left\langle\Pi_{*}(v), e_{i}\right\rangle$ where $\langle$,$\rangle denotes inner$ product. Note that $D \omega_{i}=0$ for all $i$.

For tangent vectors $v_{1}, \ldots, v_{p}$ to $F(M)$ at $z \in F(M)$, it follows from the definition of the $p$ th curvature operator that for $I=\left\{i_{1}, \ldots, i_{p}\right\}$ we have

$$
\begin{aligned}
& \theta_{I}\left(v_{1}, \ldots, v_{p}\right)=\left\langle R_{p}\left(e_{i_{1}} \wedge \cdots \wedge e_{i_{p}}\right), \Pi_{*}\left(v_{1}\right) \wedge \cdots \wedge \Pi_{*}\left(v_{p}\right)\right\rangle \\
& =\left\{\sum_{1 \leqslant j_{1}<\cdots<j_{p} \leqslant 2 n}\left\langle R_{p}\left(e_{i_{1}} \wedge \cdots \wedge e_{i_{p}}\right), e_{j_{1}} \wedge \cdots \wedge e_{j_{p}}\right\rangle\right. \\
& \left.\times \omega_{j_{1}} \wedge \cdots \wedge \omega_{j_{p}}\right\}\left(v_{1}, \ldots, v_{p}\right) .
\end{aligned}
$$

Hence, at $z=\left(m ; e_{1}, \ldots, e_{2 n}\right) \in F(M)$ and for $I=\left\{i_{1}, \ldots, i_{p}\right\}$,

(9) $\quad \theta_{I}=\sum_{1 \leqslant j_{1}<\cdots<j_{p} \leqslant 2 n}\left\langle R_{p}\left(e_{i_{1}} \wedge \cdots \wedge e_{i_{p}}\right), e_{j_{1}} \wedge \cdots \wedge e_{j_{p}}\right\rangle \omega_{j_{1}} \wedge \cdots \wedge \omega_{j_{p}}$.

Now define the subbundle $U(M)$ of $F(M)$ consisting of the unitary frames on $M$, i.e., $\quad z=\left(m: J e_{1}, \ldots, J e_{n}, e_{1}, \ldots, e_{n}\right)$ is an element of $U(M)$ if $\left\{J e_{1}, \ldots, J e_{n}, e_{1}, \ldots, e_{n}\right\}$ is an orthonormal basis of $M_{m}$. Then $U(M)$ has structure group $U(n)$ of unitary matrices on $\mathrm{C}^{n}$ considered as the subgroup of $O(2 n)$ consisting of the matrices commuting with the matrix

$$
J_{0}=\left(\begin{array}{cc}
0 & I_{n} \\
-I_{n} & 0
\end{array}\right)
$$

where $I_{n}$ is the $n \times n$ identity matrix. Note that this representation of $U(n)$ into $O(2 n)$, called the real representation of $U(n)$, is given by

$$
A+i B \rightarrow\left(\begin{array}{cc}
A & B \\
-B & A
\end{array}\right)
$$

for $A+i B \in U(n)$, where both $A$ and $B$ are real $n \times n$ matrices.

From $\S \S 3$ and 4 , Chapter IX in [14], it follows that the Riemannian connection of $M$ is a connection in $U(M)$, i.e., the connection form and curvature form $\Omega$ of the Riemannian connection of $M$ in $F(M)$, when restricted to $U(M) \subset F(M)$, take values in the Lie algebra $u(n)$ considered as its real representation in $o(2 n)$. We may 
restrict $\theta_{I}, \omega_{i}$ and all formulas above involving them to $U(M)$ without any alteration. In the following portion of the proof, we only work in $U(M)$ and treat all forms used previously as defined on $U(M)$.

Let $\mathscr{J}=\left\{J \mid J=\left\{j_{1}, \ldots, j_{p}\right\}\right.$ with $\left.1 \leqslant j_{1}<\cdots<j_{p} \leqslant 2 n\right\}$. Given $z=$ $\left(m ; e_{1^{*}}, \ldots, e_{n^{*}}, e_{1}, \ldots, e_{n}\right) \in U(M)$, where $e_{i^{*}}=J e_{i}$ for $i=1, \ldots, n$, and $J \in \mathscr{J}$, we define $E_{J}(z)=f_{j_{1}} \wedge \cdots \wedge f_{j_{p}}$ where

$$
f_{j_{k}}= \begin{cases}e_{j_{k}^{*}} & \text { if } j_{k} \leqslant n, \\ e_{j_{k}-n} & \text { if } j_{k}>n .\end{cases}
$$

For all $I, J \in \mathscr{J}$ we define $C^{\infty}$ functions $R_{I, J}$ on $U(M)$ by $R_{I, J}(z)=\left\langle R_{p}\left(E_{I}\right), E_{J}\right\rangle$. $J \in \mathscr{J}$ is said to be holomorphic if $E_{J}(z)$ is a holomorphic $p$-plane for all $z \in U(M)$. Now let $\omega_{J}=\omega_{j_{1}} \wedge \cdots \wedge \omega_{j_{p}}$ for all $J \in \mathscr{J}$. Then on $U(M)$ for $I \in \mathscr{J}$, formula (9) becomes

$$
\theta_{I}=\sum_{J \in \mathscr{g}} R_{I, J} \omega_{J}
$$

Let $H$ be the $C^{\infty}$ function on $U(M)$ obtained by taking the $C^{\infty}$ function $H^{\prime}$ on $M$ representing the pointwise constant $p$ th holomorphic sectional curvature and composing it with the projection $\Pi: U(M) \rightarrow M$. For $p=2 k$, let $I_{0}=$ $\{1,2, \ldots, k, n+1, n+2, \ldots, n+k\} \in \mathscr{J}$. Then formulas (8) and (10) give for all $z \in U(M)$

$$
\begin{aligned}
\theta_{I_{0}}(z)= & H(z) \omega_{I_{0}}(z)+\frac{1}{2} H(z) \sum_{J \in A} \omega_{J}(z) \\
& +0 \cdot \sum_{J \in B} \omega_{J}(z)+\sum_{J \in C} R_{I_{0}, J}(z) \omega_{J}(z),
\end{aligned}
$$

where

$$
\begin{gathered}
A=\left\{J \in \mathscr{J} \mid J \neq I_{0}, J \text { is holomorphic, and } J \cap I_{0}\right. \text { is } \\
\text { holomorphic of order } p-2\}, \\
B=\left\{J \in \mathscr{J} \mid J \text { is not holomorphic, } J \cap I_{0}\right. \text { contains a } \\
\text { holomorphic subset of order } p-2\}, \\
C=\left\{J \in \mathscr{J} \mid J \cap I_{0}\right. \text { does not contain a holomorphic } \\
\text { subset of order } p-2\} .
\end{gathered}
$$

Note that $\mathscr{J}=A \cup B \cup C \cup\left\{I_{0}\right\}$. Thus $D \omega_{J}=0$ for all $J \in \mathscr{J}$, and (11) gives

$$
0=D \theta_{I_{0}}=D H \wedge\left[\omega_{I_{0}}+\frac{1}{2} \sum_{J \in A} \omega_{J}\right]+\sum_{J \in C}\left[\left(D R_{I_{0}, J}\right) \wedge \omega_{J}\right] .
$$

Since $H$ is constant on the fibers of $U(M), d H$ is a horizontal 1-form and equals $D H$. Letting $d H=\sum_{i=1}^{2 n} H_{i} \omega_{i}$ and $D R_{I_{0}, J}=\sum_{k=1}^{2 n} R_{I_{0}, J, k} \omega_{k}$ for $C^{\infty}$ functions $H_{i}, R_{I_{1}, J, k}$ on $U(M),(12)$ thus becomes

$$
\begin{aligned}
0= & \sum_{i \notin I_{0}} H_{i} \omega_{i} \wedge \omega_{I_{0}}+\frac{1}{2} \sum_{J \in A}\left(\sum_{i \notin J} H_{i} \omega_{i} \wedge \omega_{J}\right) \\
& +\sum_{J \in C}\left(\sum_{k \notin J} R_{I_{0}, J, k} \omega_{k} \wedge \omega_{J}\right) .
\end{aligned}
$$


It follows from the definitions of $A$ and $C$ that the intersection of $\left\{\omega_{i} \wedge \omega_{I_{0}} \mid i \notin I_{0}\right\}$ and $\left\{\omega_{i} \wedge \omega_{J} \mid i \notin J, J \in A \cup C\right\}$ is empty. Hence (13) implies that $H_{i} \equiv 0$ for each $i \notin I_{0}$ and $d H=\sum_{i \in I_{0}} H_{i} \omega_{i}$.

Let $I_{r}=\{1,2, \ldots, \bar{r}, \ldots, k, k+1, n+1, \ldots, n+r, \ldots, n+k, n+k+1\}$ for $r$ $=1, \ldots, k$ where ${ }^{-}$over a number indicates that the number is to be deleted. Then repeating the procedure used above for $I_{0}$ on each $I_{r}$ shows that $H_{i} \equiv 0$ for each $i \notin I_{r}, r=0,1, \ldots, k$. Thus $H_{i} \equiv 0$ for all $i$ and $d H \equiv 0$. Hence from the definition of $H, d H^{\prime} \equiv 0$, and $H^{\prime}$ is a constant function on $M$ since $M$ is connected.

5. Constant zero holomorphic sectional curvature. In this section we will examine the consequences of constant zero $p$ th holomorphic sectional curvature on a Kähler manifold $M$. We need the following lemmas.

LEMMA 5.1. Let $V$ be a real inner product space of dimension $2 n$ with a complex structure $J$ and Hermitian inner product. Let $T$ be a $(2 n-2) n d$ order curvature operator on $V$ satisfying the Bianchi identity, $T=T \circ J=J \circ T$, and having constant holomorphic sectional curvature $H$. Let $\left\{e_{1}, e_{1^{*}}, \ldots, e_{n}, e_{n^{*}}\right\}$ be an orthonormal oriented basis of $V$, where $e_{i^{*}}=J e_{i}$ for all $i$, and

$$
B=\left\{e_{i_{1}} \wedge \cdots \wedge e_{i_{2 n-2}} \mid 1 \leqslant i_{1}<\cdots<i_{2 n-2} \leqslant n^{*}, 1<1^{*}<\cdots<n<n^{*}\right\} .
$$

Then for $Q \in B$, formula (6) holds. In particular, if $H=0$, then $T$ is identically zero.

Proof. Let $* 2, *(2 n-2)$ be the respective Hodge star operators. Then applying Corollary 1.10 to $*(2 n-2) T^{*} 2$ and using Corollary 1.4 together with Lemma 1.7 we obtain the above result.

Next we restate the corollary in $\S 2$ of Thorpe [26] as follows.

LEMMA 5.2. Let $M$ be a Riemannian manifold of dimension $n$, and $p, q$ be positive even integers with $p+q \leqslant n$. Let $\left\{e_{1}, \ldots, e_{p+q}\right\}$ be an orthonormal basis for a $(p+q)$-plane $P$ at $m \in M$, and $B=\left\{e_{i_{1}} \wedge \cdots \wedge e_{i_{p}} \mid 1 \leqslant i_{1}<\cdots<i_{p} \leqslant p+q\right\}$. Then

$$
R_{p+q}(P)=\frac{p ! q !}{(p+q) !} \sum_{Q \in B} R_{p}(Q) \wedge R_{q}\left(Q^{\perp}\right),
$$

where $Q^{\perp}$ is the oriented orthogonal complement of $Q$ in $P$, and $R_{p}, R_{q}, R_{p+q}$ are the pth, qth, $(p+q)$ th curvature operators of $M$, respectively.

THEOREM 5.3. Let $M$ be a Kähler manifold of real dimension $2 n$ for $n \geqslant 2$, and $p$ be an even integer with $2 \leqslant p<2 n$. Let the pth holomorphic sectional curvature of $M$ at $m_{0} \in M$ be identically zero. Then the qth holomorphic sectional curvature of $M$ at $m_{0}$ is identically zero for all even $q \geqslant p$. In particular, at $m_{0} \in M$ the Lipschitz-Killing curvature $K\left(m_{0}\right)=0$, and $M$ is $(2 n-2)$-flat at $m_{0}$.

PRoof. Throughout the proof we work only at $m_{0} \in M$. Let $R_{r}$ be the $r$ th curvature operator of $M$ for even integers $r$ with $2 \leqslant r<2 n$.

In order to prove the theorem, it is sufficient to show that constant zero $r$ th holomorphic sectional curvature of $M$ implies constant zero $(r+2)$ nd holomorphic sectional curvature of $M$ for an even integer $r$ with $2 \leqslant r<2 n$. Note that constant 
zero $(2 n-2)$ nd holomorphic sectional curvature of $M$ implies that $M$ is $(2 n-2)$ flat, by Lemma 5.1 .

Assume constant zero $r$ th holomorphic sectional curvature of $M$ for an even integer $r$ with $2 \leqslant r<2 n$. Let $P$ be a holomorphic $(r+2)$-plane with an orthonormal basis $\left\{e_{1}, e_{1^{*}}, \ldots, e_{s}, e_{s^{*}}\right\}$ where $e_{i^{*}}=J e_{i}$ for all $i$ and $2 s=r+2$. Then $P=e_{1} \wedge e_{i^{*}} \wedge \cdots \wedge e_{s} \wedge e_{s^{*}}$. Extend this basis of $P$ to an orthonormal basis $\left\{e_{1}, e_{1^{*}}, \ldots, e_{n}, e_{n^{*}}\right\}$ of $M_{m_{0}}$, where $e_{i^{*}}=J e_{i}$ for all $i$. Let $B=\left\{e_{i_{1}} \wedge \cdots \wedge e_{i_{r}} \mid 1 \leqslant\right.$ $\left.i_{1}<\cdots<i_{r} \leqslant s^{*}\right\}, B^{\prime}=\left\{e_{i_{1}} \wedge \cdots \wedge e_{i_{r}} \mid 1 \leqslant i_{1}<\cdots<i_{r} \leqslant n^{*}\right\}$, and $B^{\prime \prime}=$ $\left\{e_{i_{1}} \wedge e_{i_{2}} \mid 1 \leqslant i_{1}<i_{2} \leqslant n^{*}\right\}$ where $1<1^{*}<\cdots<n<n^{*}$. Then $B, B^{\prime}, B^{\prime \prime}$ are orthonormal bases of $\Lambda^{r}(P), \Lambda^{r}\left(M_{m_{0}}\right)$, and $\Lambda^{2}\left(M_{m_{0}}\right)$, respectively.

Let $\Pi_{p}: \Lambda^{r}\left(M_{m_{0}}\right) \rightarrow \Lambda^{r}(P)$ be the orthogonal projection map. Note that $J \circ \Pi_{P}$ $=\Pi_{P} \circ J$ since $P$ is holomorphic, and $J\left(\Lambda^{r}(P)\right)=\Lambda^{r}(P)$. We can define an $r$ th-order curvature operator on $P$ by $R_{r}^{\prime}=\Pi_{P} \circ\left(\left.R_{r}\right|_{\Lambda^{r}(P)}\right)$. Then $R_{r}^{\prime}$ satisfies the Bianchi identity, $R_{r}^{\prime}=J \circ R_{r}^{\prime}=R_{r}^{\prime} \circ J$, and $R_{r}^{\prime}$ has constant zero holomorphic sectional curvature since $R_{r}$ does. From Lemma 5.1 it follows that $R_{r}^{\prime}$ is identically zero. Hence for all $Q \in B$ we have that $\Pi_{P}\left(R_{r}(Q)\right)=0$.

By Lemma 5.2 and formula (14),

$$
R_{r+2}(P)=\frac{r ! 2 !}{(r+2) !} \sum_{Q \in B} R_{r}(Q) \wedge R_{2}\left(Q^{\perp}\right)
$$

For each $Q \in B$ we have

$$
\begin{gathered}
R_{r}(Q)=\sum_{Q^{\prime} \in B^{\prime}}\left\langle R_{r}(Q), Q^{\prime}\right\rangle Q^{\prime}, \\
R_{2}\left(Q^{\perp}\right)=\sum_{Q^{\prime \prime} \in B^{\prime \prime}}\left\langle R_{2}\left(Q^{\perp}\right), Q^{\prime \prime}\right\rangle Q^{\prime \prime},
\end{gathered}
$$

so

$$
R_{r}(Q) \wedge R_{2}\left(Q^{\perp}\right)=\sum_{Q^{\prime} \in B^{\prime}, Q^{\prime \prime} \in B^{\prime \prime}}\left[\left\langle R_{r}(Q), Q^{\prime}\right\rangle\left\langle R_{2}\left(Q^{\perp}\right), Q^{\prime \prime}\right\rangle\left(Q^{\prime} \wedge Q^{\prime \prime}\right)\right] .
$$

Letting $B^{\prime \prime \prime}=B^{\prime \prime} \cap \Lambda^{2}(P)$ and using the above with formula (15), we obtain

$$
\left\langle R_{r+2}(P), P\right\rangle=\frac{r ! 2 !}{(r+2) !} \sum_{\substack{Q . Q^{\prime} \in B^{\prime} \\ Q^{\prime \prime} \in B^{\prime \prime \prime}}}\left\langle R_{r}(Q), Q^{\prime}\right\rangle\left\langle R_{2}\left(Q^{\perp}\right), Q^{\prime \prime}\right\rangle\left\langle Q^{\prime} \wedge Q^{\prime \prime}, P\right\rangle .
$$

But $\Pi_{P}\left(R_{r}(Q)\right)=0$ for all $Q \in B$, i.e., $\left\langle R_{r}(Q), Q^{\prime}\right\rangle=0$ for all $Q, Q^{\prime} \in B$. Hence $\left\langle R_{r+2}(P), P\right\rangle=0$, so that constant zero $r$ th holomorphic sectional curvature of $M$ implies constant zero $(r+2)$ nd holomorphic sectional curvature of $M$.

The following Theorem is a corollary of Theorem 5.3.

THEOREM 5.4. Let $M$ be a compact Kähler manifold of real dimension $2 n$ for $n \geqslant 2$. For an even integer $p$ with $2 \leqslant p<2 n$, let the pth holomorphic sectional curvature of $M$ be identically zero. Then the Euler-Poincare characteristic $\chi(M)=0$ and the $(n-1)$ st Chern class $\gamma_{n-1}$ is zero. 
Proof. By Theorem 5.3 we know that the Lipschitz-Killing curvature $K$ of $M$ is identically zero, and so the generalized Gauss-Bonnet theorem gives $\chi(M)=0$.

Also by Theorem 5.3 we know that $M$ is $(2 n-2)$-flat, i.e., $R_{2 n-2}$ is identically zero. Hence from Theorem 1.11 and formula (7), it follows that $\gamma_{n-1}$ is zero.

Our last theorem involves the vanishing of Chern classes for a compact Kähler manifold $M$.

THEOREM 5.5. Let $M$ be a compact Kähler manifold of real dimension $2 n$ for $n \geqslant 2$. For an even integer $p$ with $2 \leqslant p<2 n$, let $R_{p}(Q)=0$ for all holomorphic $p$-planes $Q$, where $R_{p}$ is the pth curvature operator of $M$. Then the Chern classes $\gamma_{q}$ of $M$ are all zero for all $q \geqslant p / 2$.

Proof. By Theorem 1.11 and formula (7), it is sufficient to show that if $R_{r}(Q)=0$ for all holomorphic $r$-planes $Q$ with $r$ an even integer such that $2 \leqslant r<2 n$, then $R_{r+2}\left(Q^{\prime}\right)=0$ for all holomorphic $(r+2)$-planes $Q^{\prime}$.

Let $P$ be a holomorphic $(r+2)$-plane with orthonormal basis $\left\{e_{1}, e_{1^{*}}, \ldots, e_{s}, e_{s^{*}}\right\}$ where $e_{i^{*}}=J e_{i}$ for all $i$ and $2 s=r+2$. Then $P=e_{1} \wedge e_{1^{*}} \wedge \cdots \wedge e_{s} \wedge e_{s^{*}}$. Let $B=\left\{e_{i_{1}} \wedge \cdots \wedge e_{i_{r}} \mid 1 \leqslant i_{1}<\cdots<i_{r} \leqslant s^{*}\right.$ with $\left.1<1^{*}<\cdots<s<s^{*}\right\}$. Then by Lemma 5.2 , formula (15) holds.

Assume that $R_{r}(Q)=0$ for all holomorphic $r$-planes $Q$, and let

$$
Q^{\prime}=X_{1} \wedge J X_{1} \wedge \cdots \wedge X_{t} \wedge J X_{t} \wedge Y \wedge Z
$$

where $2 t=r-2$. Then

$$
\begin{aligned}
0= & R_{r}\left(X_{1} \wedge J X_{1} \wedge \cdots \wedge X_{t} \wedge J X_{t} \wedge(Y-J Z) \wedge(J Y+Z)\right) \\
= & R_{r}\left(X_{1} \wedge J X_{1} \wedge \cdots \wedge X_{t} \wedge J X_{t} \wedge Y \wedge J Y\right) \\
& +R_{r}\left(X_{1} \wedge J X_{1} \wedge \cdots \wedge X_{t} \wedge J X_{t} \wedge Z \wedge J Z\right) \\
& +2 R_{r}\left(X_{1} \wedge J X_{1} \wedge \cdots \wedge X_{t} \wedge J X_{t} \wedge Y \wedge Z\right) \\
= & 2 R_{r}\left(Q^{\prime}\right) .
\end{aligned}
$$

Hence $R_{r}\left(Q^{\prime}\right)=0$ for any $r$-plane $Q^{\prime}$ containing a holomorphic $(r-2)$-subplane. From this, it is clear that $R_{r}(Q)=0$ for all $Q \in B$ since each of these contains a holomorphic $(r-2)$-subplane. Thus formula (15) implies that $R_{r+2}(P)=0$.

\section{REFERENCES}

1. R. L. Bishop and S. I. Goldberg, Some implications of the generalized Gauss-Bonnet theorem, Trans. Amer. Math. Soc. 11 (1964), 508-535.

2. On the second cohomology group of a Kähler manifold of positive curvature, Proc. Amer. Math. Soc. 16 (1965), 119-122.

3. S. Block and D. (iesieker, The positivity of the Chern classes of an ample vector bundle, Invent. Math. 12 (1971), 112-117.

4. J. P. Bourguignon and H. Karcher, Curtature operators: pinching estimates and geometric examples, Ann. Sci. École Norm. Sup. 11 (1978), 71-92.

5. S. S. Chern, On the curtature and characteristic classes of a Riemannian manifold, Abh. Math. Sem. Univ. Hamburg 20 (1956), 117-126.

6. Y. K. Cheung and C. C. Hsiung. Curvature and characteristic classes of compact Riemannian manifolds, J. Differential Geom. 1 (1967), 89-97.

7. R. (jeroch, Positive sectional curtature does not imply positive Gauss-Bonnet integrand, Proc. Amer. Math. Soc. 54 (1976), 267-270. 
8. A. Gray, A generalization of F. Schur's theorem, J. Math. Soc. Japan 21 (1969), 454-457.

9. , Some relations between curvature and characteristic classes, Math. Ann. 184 (1970), $257-267$.

10. Chern numbers and curvature, Amer. J. Math. 100 (1978), 463-476.

11. W. V. Hodge and D. Pedoe, Methods of algebraic geometry, Cambridge Univ. Press, Cambridge, 1947.

12. D. L. Johnson, Curvature and Euler characteristic for six-dimensional Kähler manifolds, Illinois J. Math. 28 (1984), 654-675.

13. P. Klembeck, On Geroch's counterexample to the algebraic Hopf conjecture, Proc. Amer. Math. Soc. 59 (1976), 334-336.

14. S. Kobayashi and K. Nomizu, Foundations of differential geometry, Vol. I, 1963; Vol. II, 1969, Wiley, New York.

15. R. S. Kulkarni, On the Bianchi identities, Math. Ann. 199 (1972), 175-204.

16. D. Meyer, Sur les variétés Riemanniennes á opérateur de courbure positif, C.R. Acad. Sci. Paris Ser. A-B 272 (1971), 482-485.

17. S. Mori, Projective manifolds with ample tangent bundles, Ann. of Math. (2) 110 (1979), 590-606.

18. A. M. Naveira, Charactérisation des variétés à courbures sectionnelles holomorphes généralisées constantes, J. Differential Geom. 9 (1974), 55-60.

19. On the higher order sectional curvatures, Illinois J. Math. 19 (1975), 165-172.

20. I. M. Singer and J. A. Thorpe, The curvature of 4-dimensional Einstein spaces, Global Analysis, Papers in Honor of K. Kodaira, Univ. of Tokyo Press and Princeton Univ. Press, 1969, pp. 355-365.

21. Y. T. Siu and S. T. Yau, Compact Kähler manifolds of positive bisectional curvature, Invent. Math. 59 (1980), 189-204.

22. A. Stehney, Courbure d'ordre p et les classes de Pontrjagin, J. Differential Geom. 8 (1973), 125-134.

23. __ Extremal sets of pth sectional curvature, J. Differential Geom. 8 (1973), 383-400.

24. J. A. Thorpe, Sectional curvature and characteristic classes, Ann. of Math. (2) 80 (1965), 429-443.

25. On the curvature of Riemannian manifolds, Illinois J. Math. 10 (1966), 412-417.

26. __ Some remarks on the Gauss-Bonnet integral, J. Math. Mech. 18 (1969), 779-786.

27. _ On the curvature tensor of a positively curved 4-manifold, Proc. 13th Biennial Sem. Canad.

Math. Congress, Vol. 2, 1971, pp. 156-159.

Department of Mathematics, Lehigh University, Bethlehem, Pennsylvania 18015

Department of Mathematics, Eastern Michigan University, Ypsilanti, Michigan 48197 